\title{
Rehabilitación Cognitiva y Estimulaciones Naturales en Mayores de 55 Años con Deterioro Cognitivo Leve e Hipertensión
}

\section{Cognitive Rehabilitation and Natural Stimulations in Over 55 Years with Mild Cognitive Impairment and Hypertension}

\author{
Dides Iliana Hernández Silvera y Eduardo Miguel Leonardelli ${ }^{1}$ \\ Facultad de Psicología y Psicopedagogía, Pontificia Universidad Católica Argentina
}

\begin{abstract}
La prevalencia creciente de deterioro cognitivo leve (DCL) comórbido con hipertensión arterial (HTA) exige revisión y mejora de las intervenciones disponibles. El objetivo del estudio fue comparar el efecto en las funciones cognitivas de la rehabilitación cognitiva (RC) y otras formas de estimulación. Con un diseño cuasi experimental, se evaluaron cuatro estímulos naturales (actividad física, actividades intelectuales domiciliarias, hobbies, trabajo) de 128 pacientes mayores a 55 años, hipertensos y con deterioro de por lo menos dos áreas de desempeño cognitivo y con deterioro vascular, seleccionados mediante un muestreo por conveniencia. Cuarenta y cuatro de ellos participaron voluntariamente en sesiones de RC. Se compararon los efectos de la RC y de los estímulos naturales sobre cinco funciones cognitivas, tres relativas al lenguaje (denominativo, comprensivo y fluencia verbal) memoria global y atención; valoradas desde el ACE Test (dominios atención, memoria, fluencia verbal y lenguaje), Token Test. La comparación se realizó mediante un modelo multivariado para medidas repetidas con control de variables eventualmente confundidoras. Los principales resultados fueron: menor edad y mayor trayectoria académica predicen un mejor nivel inicial en las funciones evaluadas. La RC se asocia a ganancias en atención, memoria y fluencia verbal. Las actividades intelectuales domiciliarias también muestran efectos positivos. Las mayores ganancias se registran entre quienes tenían mayor deterioro cognitivo y menor trayectoria académica. La postulación de la reserva cognitiva restante sintetizaría estos fenómenos. La principal conclusión es que la RC es la estimulación prioritaria, seguida por actividades intelectuales domiciliarias, para el mantenimiento o recuperación parcial de funciones cognitivas en pacientes con DCL e HTA.
\end{abstract}

Palabras clave: hipertensión, deterioro cognitivo, rehabilitación cognitiva, remisión, estímulo natural

\begin{abstract}
The increasing prevalence of mild cognitive impairment (MCI) comorbid with arterial hypertension requests the revision and enhancement of available interventions. The goal of this study was to compare the effect of cognitive rehabilitation (CR) and other forms of stimulation on cognitive functions. Using a quasi-experimental design and a convenience sampling, the reception of four natural stimuli (physical and intellectual activities, hobbies, work) was collected from 128 hypertensive patients aged over 55 years with cognitive (at least two areas) and vascular impairment. Forty-four of them willingly participated in CR sessions. The effects of CR and natural stimuli on five cognitive functions were compared, three related to language (comprehensive, denominative, and fluent language), global memory and attention, assessed with the ACE Test (attention, memory, verbal fluency and language domains). Multivariate modeling analysis for repeated measures was used, controlling for potentially confounding variables. Main results show that younger age and higher academic trajectory predict better initial levels of the evaluated functions. CR is associated with gains in attention, memory and verbal fluency. Written home cognitive activities show also positive effects. The larger gains are observed in participants with higher cognitive impairment and lower academic trajectories. The postulate of the remaining cognitive reserve would synthesize these phenomena. The study concludes that CR is the preferred form of stimulation, followed by home intellectual exercises for the maintenance and partial recovery of cognitive functions in patients with mild cognitive impairment and hypertension.
\end{abstract}

Keywords: hypertension, cognitive impairment, cognitive rehabilitation, remission, natural stimulation

Dides Iliana Hernández Silvera (iD https://orcid.org/0000-0001-7759-516X

Eduardo Miguel Leonardelli (iD https://orcid.org/0000-0002-3607-076X

Los autores agradecen al Dr. Facundo Manes por su consentimiento en el uso del ACE Test. Conflicto de intereses: La primera autora se dedica en parte a la rehabilitación cognitiva y su centro de atención ha provisto los datos que aquí se analizan.

La correspondencia relativa a este artículo debe ser dirigida a Dides Iliana Hernández Silvera, Facultad de Psicología y Psicopedagogía, Pontificia Universidad Católica Argentina, Alicia Moreau de Justo 1500, PB, Buenos Aires, Argentina. Email: hernandezsilvera@uca.edu.ar 
HERNÁNDEZ-SILVERA Y LEONARDELLI

\section{Los Desafíos del Envejecimiento y la Trasformación de los Entornos}

Hernández Silvera (2009) y Bartoloni (2019) han descrito cómo en las últimas décadas los cambios en la demografía obligan a prestar mayor atención a las necesidades de los adultos mayores. El envejecimiento de la población plantea diversos retos a las familias, a las comunidades y a las sociedades, en aspectos como el crecimiento económico, la seguridad económica en la vejez, la organización de los sistemas de atención a la salud y la solidez de los sistemas de apoyo familiar. Junto con el aumento de la expectativa de vida, han aumentado la prevalencia en hipertensión y accidentes cerebrovasculares, los déficits y deterioros cognitivos (Garre-Olmo, 2018), profundizando los problemas relativos a la atención y calidad de vida de los adultos mayores, los costos sanitarios y la carga de familias y cuidadores. Ello pone en cuestión la eficacia del sistema y urge el desarrollo de medidas preventivas adecuadas que permitan un envejecimiento satisfactorio, encaminadas a promover el bienestar de las generaciones presentes y futuras (División de Población, 2014, Capítulo VII: Envejecimiento de la población, pp. 24-26). Estas circunstancias demandan modificaciones en el entorno macro y microsistémico tendientes a disminuir la prevalencia de enfermedades incapacitantes con deterioro cognitivo y atemperar sus efectos, a través de la implementación de estrategias, tales como talleres por niveles de deterioro y trabajo individual para quienes requieren un encuentro personalizado en rehabilitación cognitiva ( $\mathrm{RC})$.

El presente contexto de pandemia de COVID-19 afecta adicionalmente, directa e indirectamente, a la salud de las personas mayores (Schapira et al., 2020). Más allá de la amenaza a la supervivencia, Schapira et al. (2020) han resaltado que las personas mayores, por el aislamiento preventivo, se ven particularmente privadas de la interacción con el afuera y de estímulos sensoriales. Se ha aprendido, por otra parte, a hacer frente a tal deprivación mediante el uso de las tecnologías de la información y la comunicación: mensajes de WhatsApp y sesiones de Zoom, entre otras. Así, hay pacientes con deterioro cognitivo que mediante la tecnología se insertan en una verdadera realidad virtual, con la consecuente reformulación terapéutica a distancia. Todo esto constituye una ocasión propicia para la revaluación y reformulación de las propuestas terapéuticas a distancia, en neurodiagnóstico y RC, ampliando el horizonte de estrategias a incorporar y potenciando la duración de las intervenciones.

\section{El Deterioro Cognitivo Leve}

Entre los cambios en las funciones cognitivas asociados al proceso de envejecimiento se destacan la merma en memoria, atención, velocidad de procesamiento de la información, funciones visoperceptivas, lenguaje y funciones ejecutivas (Luque \& Gonzalez Verheust, 2012). Forlenza et al. (2013) afirman que un sujeto con síntomas compatibles con deterioro cognitivo (DC) podría avanzar hacia el desarrollo de algún tipo de demencia, permanecer estable e, incluso, con la intervención adecuada, retomar su funcionamiento cognitivo normal. Consecuentemente, Bruna et al. (2011) subrayan la importancia de valorar los cambios cognitivos, más allá del propio proceso de envejecimiento.

A su vez, descriptivamente, se ha buscado distinguir y delimitar conceptos para precisar las formas que toman las disfuncionalidades cognitivas. Así, la American Psychiatric Association (2014), con su Manual Diagnóstico y Estadístico de los Trastornos Mentales DSM-5, ha colaborado notoriamente en la difusión del concepto de deterioro cognitivo leve (DCL), el que se distingue del DC mayor. El DSM-5 describe y ejemplifica, desde su perspectiva operativa, las formas que toma la estructura sindrómica del DCL en distintos dominios cognitivos: atención compleja, función ejecutiva, aprendizaje y memoria, lenguaje, habilidades perceptuales y reconocimiento social. También incluye al DCL entre los criterios diagnósticos de los trastornos neurocognitivos leves.

Arroyo-Anlló et al. (2012) sugieren que las personas con DCL tienen mayor riesgo de desarrollar demencia y que habría una urgente necesidad de desarrollar intervenciones que puedan prevenir o retrasar el inicio del deterioro en estos grupos. Campbell et al. (2013) afirman que la progresión del DC compartiría con la demencia factores de riesgo, como la edad, el sexo, el nivel educativo, la carga genética y la presencia de trastornos depresivos.

Hernández Silvera (2009) expone que una intervención precoz puede mantener la funcionalidad de la persona durante mayor tiempo o enlentecer el proceso de DC. Se han buscado, por lo tanto, intervenciones orientadas a que las personas con DC puedan mantener o retomar de manera autoválida y segura sus actividades cotidianas y mejorar, en definitiva, su calidad de vida. Bruna et al. (2011) expresan la necesidad 
de revisar sistemáticamente las formas naturales de estimulación del funcionamiento cognitivo que, dados su difusión y bajo costo, permitirían contar con intervenciones más accesibles.

Así, Calero Morales et al. (2016) establecen que las actividades físico-deportivas y de recreación contribuyen a regular el proceso de forma positiva, dado que, entre otros aspectos, incrementan la percepción de competencia y de relación con el medio. También se ha afirmado que la realización de acciones vinculadas con un estilo de vida activo, esto es, actividad cognitiva (leer, pintar, escribir, hacer crucigramas), actividad física (practicar algún deporte o caminar) y actividades sociales (participación en clubes, iglesias), practicadas con regularidad, pueden retrasar la aparición del DC (Arenaza-Urquijo et al., 2011). Por último y no menos importante, Serrano et al. (2007) afirman que la inactividad laboral (jubilación) aumenta 2,5 veces el riesgo de conversión. Este cambio seguiría a la deprivación que ocurre por falta de estímulo ambiental y se relaciona, a su vez, con el DC.

Stern $(2002,2003)$ ha postulado la existencia de entidades teóricas que tratan de readaptar el cerebro de modo tal de tolerar mejor los efectos de lesiones. Una de ellas es la reserva cerebral, que tendría una forma pasiva y estaría relacionada con el tamaño y cantidad de neuronas que se mantienen intactas frente a un daño, antes de que se manifiesten síntomas clínicos. La reserva cognitiva (RCog), en cambio, sería la forma activa de afrontar las lesiones. Como tal, emplearía dos recursos: el uso de redes cerebrales o paradigmas cognitivos alternativos menos susceptibles de interrupción, preexistentes, utilizados por individuos sanos frente a tareas corrientes. El segundo recurso sería la compensación, que usa estructuras o redes cerebrales normalmente no utilizadas por individuos con cerebros intactos, para compensar el daño cerebral.

Lojo-Seoane et al. (2012) revisaron 22 investigaciones sobre RC y DCL publicadas hasta entonces y llegaron a tres conclusiones: hallaron una buena calidad de evidencia en favor de que la reserva cerebral y la RCog influyen en el retraso de la manifestación de los síntomas de DC. En segundo lugar, hay regulares evidencias de que tales retrasos pueden darse y también actuar de enmascaradores durante el período que transcurre hasta la manifestación de la demencia, aunque hay discrepancias sobre la tasa de declive que marcaría su influencia y dudas metodológicas que solo podrían despejar estudios prospectivos. Finalmente, señalan que entre las variables marcadoras de RCog evaluadas "el nivel educativo se presenta en todos los estudios como una variable sólida como indicadora de la protección contra el declive cognitivo general y también en áreas cognitivas concretas como la memoria. Además, destaca como una variable relevante desde la detección precisa del DCL y durante todo el proceso de deterioro" (p. 277). Detrás del nivel educativo, apuntan a la complejidad laboral y la participación en actividades cognitivas desde la juventud como los más importantes indicadores de RCog.

El constructo de RCog sustenta teóricamente la intervención de RC, cuya eficacia se mostrará y da cuenta, también, del estado inicial de las variables relativas a funciones cognitivas.

\section{Los Estímulos y el DCL}

Cancino y Rehbein (2016) vinculan a la edad y a la disminución de la actividad intelectual y social a partir de los 40 años con mayor DC en la tercera edad, independientemente del nivel socioeconómico y la alfabetización. Además, Portillo Barrera (2015) y Hernando-Requejo (2016) resaltan la importancia de la actividad física regular y del tratamiento de los factores de riesgo cardiovascular, como la diabetes, obesidad e hipertensión, en la disminución del riesgo de DC y de demencia. Los mismos autores sostienen que una alimentación saludable y el aprendizaje y entrenamiento cognitivo durante toda la vida reducen también el riesgo de DC.

Cancino y Rehbein (2016), a partir del estudio de sujetos de entre 60 y 89 años de edad, concluyen que los sujetos con más edad y menos años de escolarización tienen mayor riesgo de cumplir con los criterios de inclusión para el diagnóstico de DCL. Meléndez-Moral et al. (2012) afirman que los años de escolaridad tienen un correlato con el tipo de actividad laboral que desarrolla una persona y esta variable también se incluye en los modelos de predicción del DC. Así, los individuos que tienen menor nivel de educación formal y han desarrollado actividades laborales de escasa creatividad tienden a presentar modificaciones o mermas cognitivas que van más allá de lo esperado para su edad.

El DC puede ser de origen vascular. El así llamado deterioro cognitivo vascular (DCV) permite agrupar un amplio espectro de trastornos cognitivos de origen vascular con semiologías y cursos evolutivos diferentes. El DCV incluye: demencias vasculares hereditarias, demencia multinfarto, demencia post ictus y patología vascular isquémica subcortical con o sin demencia (Rodríguez García \& Rodríguez García, 2015). 
Arriola Manchola et al. (2017) plantean que, en función de su mayor efectividad, las intervenciones ante el DC requieren formas diferentes en situaciones distintas: (a) la estimulación cognitiva es una facilitación general y temática de operaciones cognitivas basadas en evocación, relación y procesamiento; (b) el entrenamiento cognitivo representa el aprendizaje o refuerzo de operaciones cognitivas específicas y (c) la $\mathrm{RC}$, por su parte, consiste en la sistematización de acciones de aprendizaje o refuerzo de operaciones cognitivas, altamente individualizada, dirigida a mantener o a recuperar capacidades funcionales o sociales relevantes para el enfermo. La efectividad de las intervenciones dependería de una serie de pasos previos a realizar, tales como una buena valoración cognitiva, un mapa de vida con aficiones y hobbies de la persona con deterioro para aprovechar al máximo sus capacidades y potenciales, la motivación y participación en actividades de diversa índole.

Por otra parte, Nevado Rey (2017) afirma el valor del ejercicio físico regular, que ayudaría a potenciar la movilidad, trabajar la respiración y mejorar significativamente los patrones cardiovasculares, evitando problemas de este tipo que pudieran hacer avanzar el DC. De la misma manera, manifiesta que las nuevas tecnologías abren las puertas al desarrollo y trabajo con programas de estimulación, a través de ordenadores, tabletas o videojuegos. Programas como Gradior y Smart Brain disponen de un apoyo empírico discreto, pendientes aún de demostrar efectividad plena en situaciones de DCL con estudios que los avalen (Arriola Manchola et al., 2017).

En este orden de ideas, el presente estudio comparó de modo controlado el impacto sobre funciones cognitivas de una propuesta (véase Procedimientos e Instrumentos) de RC con el de otras formas de estimulación natural (actividades físicas, actividades mentales domiciliarias, actividades laborales y hobbies) en mayores de 55 años con DCV leve, con hipertensión arterial (HTA).

\section{Método}

\section{Diseño}

El diseño fue cuasi experimental, de comparación antes-después en grupos naturales, según la participación en sesiones presenciales de RC y la sistematización (inclusión como variables independientes [VI]) de otras cuatro formas de estímulos cognitivos, no manipuladas en el estudio: realización de ejercicios físicos (gimnasia y/o caminatas), hobbies o actividades creativas, actividades mentales domiciliarias (crucigramas, sudoku, sopas de letras, entre otras) y trabajo remunerado. La RC y las demás formas de estímulos cognitivos no son mutuamente excluyentes y pueden coexistir en diferente número en distintos participantes. El diseño incluyó, finalmente, el control estadístico de dos covariantes: puntaje en la Escala de Isquemia de Hachinski y los años de escolaridad.

\section{Participantes}

Fueron 128 participantes seleccionados por muestreo por conveniencia de la siguiente manera: todos fueron derivados por neurólogos de la Región Metropolitana de Argentina a un consultorio privado de psicopedagogía especializado en neurodiagnóstico, para evaluación por sospecha de DC, con fines de ajuste de medicación y RC, entre 2015 y 2019. Todos los pacientes derivados (357) concurrieron con estudios complementarios, tales como pruebas de laboratorio, resonancia magnética nuclear) y/o tomografía axial computada. Para este estudio, se retuvo una muestra de 128 adultos, según cumplimiento de los siguientes criterios de inclusión-exclusión:

1. edad superior a 54 años;

2. diagnóstico de HTA con deterioro de por lo menos dos áreas de desempeño cognitivo y con deterioro vascular (DV), según los criterios NINDS-AIREN, y, para establecer sospecha clínica, puntaje en la Escala de Isquemia de Hachinski $\geq 6$;

3. ausencia de depresión, valorada a partir de la Escala de Yesavage para Depresión Geriátrica (GDS);

4. ausencia de demás patologías psiquiátricas, según el registro de seguimiento del paciente en su historia clínica y un cuestionario sociodemográfico.

Las características generales de la muestra se resumen en la Tabla 1. Las más importantes son: la edad media es de 70 años ( $D E=8$ años), 11 años de escolaridad media ( $D E=3,9$ años), 76 son mujeres y 46 optaron por participar de la RC. 
Tabla 1

Estadísticos Descriptivos de la Muestra según Diferentes Formas de Estimulación

\begin{tabular}{|c|c|c|c|c|c|c|c|c|c|c|c|c|}
\hline \multicolumn{2}{|c|}{$\begin{array}{c}\text { Eventuales variables } \\
\text { confundidoras }\end{array}$} & \multicolumn{2}{|c|}{$\begin{array}{l}\text { Gimnasia y/o } \\
\text { caminatas }\end{array}$} & \multicolumn{2}{|c|}{$\begin{array}{l}\text { Actividad } \\
\text { creativa }\end{array}$} & \multicolumn{2}{|c|}{$\begin{array}{c}\text { Crucigrama, } \\
\text { sudoku, sopa de } \\
\text { letras }\end{array}$} & \multicolumn{2}{|c|}{ Trabaja } & \multicolumn{2}{|c|}{$\begin{array}{l}\text { Sesiones de } \\
\text { rehabilitación } \\
\text { cognitiva }\end{array}$} & \multirow[t]{2}{*}{ Total } \\
\hline & & No & Sí & No & Sí & No & Sí & No & Sí & No & Sí & \\
\hline \multicolumn{2}{|l|}{$n$} & 84 & 44 & 91 & 37 & 106 & 22 & 55 & 73 & 82 & 46 & 128 \\
\hline \multirow[b]{2}{*}{ Sexo } & Fem. & 54 & 22 & 51 & 25 & $57^{*}$ & $19^{*}$ & 36 & 40 & 46 & 30 & 76 \\
\hline & Masc. & 30 & 22 & 40 & 12 & $49^{*}$ & $3^{*}$ & 19 & 33 & 36 & 16 & 52 \\
\hline \multirow{2}{*}{ Edad } & Media & $72^{*}$ & $66^{*}$ & 71 & 68 & 70 & 69 & $74^{*}$ & $66^{*}$ & 70 & 70 & 70 \\
\hline & $D E$ & 8,0 & 6,5 & 8,2 & 7,1 & 7,9 & 8,8 & 7,3 & 6,8 & 7,9 & 8,3 & 8,0 \\
\hline \multirow{2}{*}{ Años de estudio } & Media & 10 & 11 & 11 & 11 & 11 & 11 & 10 & 11 & 11 & 10 & 11 \\
\hline & $D E$ & 3,9 & 4,1 & 4,0 & 3,9 & 3,9 & 3,9 & 4,1 & 3,7 & 4,0 & 3,7 & 3,9 \\
\hline \multirow{2}{*}{ Escala de Hachinski } & Media & 9,3 & 9,5 & 9,2 & 9,7 & 9,4 & 9,4 & 9,5 & 9,3 & 9,2 & 9,6 & 9,4 \\
\hline & $D E$ & 1,9 & 2,0 & 1,9 & 1,9 & 1,9 & 1,9 & 2,2 & 1,7 & 1,9 & 1,8 & 1,9 \\
\hline
\end{tabular}

Nota. * Diferencias con $p<0,05$. Las diferencias encontradas en sexo se evaluaron con $\chi^{2}$ con corrección de continuidad para la variable crucigrama, sudoku, sopa de letras: $\chi^{2}=6,95$, $p<0,008$. Las diferencias encontradas en edad se evaluaron con prueba $t$ suponiendo varianzas diferentes para la realización de gimnasia y/o caminatas: $t(104)=4,49 ; p<0,001 ; d=0,8$, $95 \%$ IC $[3,28,6,46]$ y con prueba $t$ suponiendo varianzas iguales para la existencia de actividad laboral rentada $($ Trabaja $): t(127)=6,25 ; p<0,001, d=1,11,95 \%$ IC $[5,33,10,27]$. 


\section{Instrumentos}

En el marco de la práctica clínica referida, se empleó la batería neurodiagnóstica inicial, repetida entre 6 y 12 meses después, considerando para el presente estudio los siguientes componentes:

\section{Evaluación Clínica según los Criterios NINDS-AIREN}

Para establecer la existencia de deterioro vascular se realizó una evaluación clínica (Pohjasvaara et al., 2000), como criterio de inclusión.

\section{Escala de Yesavage para Depresión Geriátrica (GDS-15; Yesavage \& Sheikh, 1986)}

La GDS-15 rastrea síntomas cognoscitivos de un episodio depresivo mayor, durante los últimos 15 días, mediante respuestas dicotómicas $(1=s i ; 0=n o)$ a sus 15 preguntas (10 positivas y 5 negativas). Su administración toma alrededor de cinco minutos, así se reducen los problemas de fatiga y pérdida de atención frecuentes en población mayor que suele presentar algún grado de alteración cognitiva. Según Yesavage y Sheikh (1986), la puntuación media obtenida originalmente en la escala GDS-15 fue de 4,7 (DE = 3,5, rango: $0-14)$, superior en las mujeres $(5,5 ; \mathrm{DE}=3,6)$ que en los varones $(2,9 ; \mathrm{DE}=2,4)$, sin detectarse diferencias en relación a edad ni nivel de estudios. Aquí, se utilizó la adaptación a población mayor argentina de Martínez de la Iglesia et al. (2002) llamada GDL-VE.

Martínez de la Iglesia et al. (2002) reportan suficiente confiabilidad interobservador e intraobservador (Kappa $=0,655, p<0,001)$ y $0,951(p<0,001)$ respectivamente, para un punto de corte de 5 o más; la consistencia interna alcanzó un valor de KR-20 = 0,994. En cuanto a la validez convergente, Martínez de la Iglesia et al. (2002) indican que la GDS-VE alcanzó un coeficiente de correlación de Spearman de 0,618 $(p<0,001)$ al compararlo con los resultados del cuestionario de Montgomery-AsberG; en tanto presentó buena validez discriminante, al correlacionar con la obtenida con el SPMSQ-VE, con un coeficiente de correlación de Spearman de 0,235 ( $p<0,001)$.

La GDS-15 solo se usó en este estudio como criterio de exclusión, el punto de corte con mejor relación sensibilidad/especificidad ha sido el de 5 o más puntos.

\section{Historia Clínica de los Pacientes}

De las historias clínicas de los pacientes se extrajeron sexo, edad, formas de estimulación cognitiva, incluyendo actividad laboral, y la ausencia de patologías psiquiátricas concomitantes.

\section{Cuestionario Sociodemográfico}

Incluyó, entre otras variables, el historial académico y laboral y las formas de estimulación.

\section{Escala de Isquemia Hachinski (Hachinski et al., 1975)}

Se utiliza para el diagnóstico de la demencia multinfarto, contiene 13 ítems que se puntúan con 1 o 2 puntos. Valores menores identifican Enfermedad de Alzheimer, valores mayores identifican Enfermedad Vascular. La validez de esta escala ha sido investigada en varios estudios clínicos que además estimaron una especificidad y sensibilidad del orden del 70-80\% (Diaz et al., 1991; Hachinski et al., 1975, 2006; Pantoni \& Inzitari, 1993; Ye et al., 2019). En este estudio se usó como punto de corte por lo menos 6 puntos para la identificación de la DV, conforme a Thomas Carazo y Nadal Blanco (2001).

Además de ser criterio de inclusión, el puntaje de la Escala de Isquemia de Hachinski fue incluido en los análisis como variable controlada.

\section{Addenbrook's Cognitive Examination (Test ACE)}

Propuesto originalmente como test de cribado (Mathuranath et al., 2000), con el objetivo de valorar la calidad de las medidas de la versión española del ACE por medio del modelo de Rasch y comprobar su utilidad diagnóstica como herramienta de screening en demencias. Con 15-20 minutos de administración, superó en su capacidad discriminante de demencia frontotemporal y demencia tipo Alzheimer al MiniMental State Examination. Sarasola et al. (2005) llevó a cabo una adaptación al español en España, con una 
muestra semejante el presente estudio, registrando un alfa de Cronbach de 0,81 , y alcanzando, mediante el coeficiente (VLOM), fluidez verbal+lenguaje / orientación+recuerdo diferido, una sensibilidad del 95,5\% para detectar demencia frontotemporal y una especificidad del 40,7\%. El Addenbrooke's Cognitive Examination (ACE) batería breve de cribado en demencias tiene un estudio de validación en Argentina en poblaciones de alta (AE) y baja educación (BE) (Sarasola et al., 2005; Souza \& Vivas, 2017). La versión argentina del ACE evalúa 6 dominios cognitivos. El puntaje máximo es de 100: orientación (10), atención (8), memoria (35), fluencia verbal (14), lenguaje (28) y habilidades visuoespaciales (5). El puntaje de corte para BE es de 68/100 y el de AE 86/100. A su vez, permite calcular los 30 puntos del Mini Mental State, ya que están incluidos en el cuestionario.

Si bien no se esperan modificaciones en el diagnóstico de DCL, se considera que el Test ACE posee una sensibilidad del 92\% (García-Caballero et al., 2006) para la detección de modificaciones en algunos de los seis dominios cognitivos seleccionados en función de la especificidad de la intervención: Orientación, Atención, Memoria, Lenguaje, Fluencia Verbal y Habilidades Visuoespaciales. Para el presente, estudio se usaron Atención, Memoria, Lenguaje y Fluencia Verbal como variables dependientes (VD).

\section{Test Token}

El Test Token está orientado a evaluar lenguaje comprensivo receptivo para oraciones extensas, mediante respuestas a órdenes de complejidad creciente. Su versión original (De Renzi \& Vignolo, 1962) contenía 62 órdenes. Sin embargo, existen distintas versiones que usan un número menor de órdenes: 39 (Spellacy \& Spreen, 1969), 36 (De Renzi \& Faglioni, 1978) o 16 (van Harskamp \& van Dongen, 1977). Todas ellas han mostrado niveles de confiabilidad satisfactorios, evaluados con las variantes 20 o 21 del Coeficiente Kuder-Richardson.

En esta investigación se usó la versión del Test Token de 36 ítems (De Renzi \& Faglioni, 1978), sensible para diagnóstico de trastornos de comprensión del lenguaje, al incluir series de elementos y la intervención de nexos que requieren seguimiento y comprensión ("Toque el círculo rojo o el azul", "Toque el círculo rojo con el azul", "Toque el círculo rojo y el azul"). Las puntuaciones de esta versión determinan una especificidad de 90\% para el diagnóstico de afasia. Este puntaje actuó como VD en los análisis.

\section{La Intervención}

El dispositivo de RC (implementado de 6 a 12 meses) consistió en varias posibilidades, según sugerencias terapéuticas y decisiones personales de los médicos intervinientes y de los mismos pacientes: sesiones individuales semanales, uso de plataforma, cuaderno de ejercicios semanales extrasesiones. Las sesiones individuales implicaban la concurrencia semanal a realizar en el consultorio ejercicios escritos o en computador (PC) con acompañamiento terapéutico con valoración inmediata del plan de acción. Este plan indicaba las funciones que serían reforzadas mediante programas específicos, ya que se pretendía multiestimular diferentes áreas cerebrales. Por lo antes mencionado, en cada sesión se hizo una valoración de estado.

Quienes realizaban actividad en el consultorio podían, además, ingresar a una plataforma virtual gratuita. La plataforma contenía pruebas generadas por el terapeuta administrador, juegos que proporcionaban un entrenamiento funcional y enlaces a sitios externos. La plataforma tenía un uso muy similar a otras plataformas sociales en la red, se organizó en cinco niveles de dificultad y servía de apoyo diario complementario. Quienes no manejaban PC o internet o no tenían deseos ni acceso fácil podían complementar su entrenamiento domiciliario con cuaderno de ejercicios o con estrategias de escritas. En este caso, hubo quienes decidieron solamente concurrir al consultorio sin efectuar actividades de refuerzo. Las estrategias escritas se basaron, en su mayoría, en actividades para desarrollar lenguaje, fluencia verbal, atención, memoria y, en menor medida, visuoespaciales, percepción y lógica. La capacidad eficiente y flexible de cada sujeto, que permitía optimizar su rendimiento cognitivo, fue revisada en cada sesión individual en el consultorio.

De la misma manera, otros pacientes prefirieron realizar gimnasia (caminatas, bicicleta), actividades creativas (hobbies, como artesanías, bordado, plegado de papel y tallado en madera), continuar con actividades que ya realizaban antes, como sudokus, sopas de letras o crucigramas, según datos aportados por los sujetos o sus familiares acompañantes al momento de la segunda evaluación (post). 


\section{Procedimiento}

La batería neurodiagnóstica fue administrada a los pacientes derivados antes de la intervención de RC según criterio médico. Su administración tomó una hora y media, sin registro de agotamiento. Ella fue aplicada por una especialista en Neurociencias Cognitivas, Psicogerontología y Demencias, en un centro de atención a pacientes derivados para el estudio neurocognitivo. Su segunda administración (retest) tuvo lugar entre 6 y 12 meses luego de la primera.

Todos los pacientes derivados firmaron un consentimiento informado para fines de investigación. El orden de la aplicación de los instrumentos fue: Batería Global ACE Test, Test Token, Test Boston Adaptación a Adultos, Trail Making (Test A y B) y la GDS-VE.

De acuerdo al neurodiagnóstico, se sugirieron o no instancias de RC.

\section{Análisis de Datos}

Las diferencias entre los puntajes postratamiento y pretratamiento se calcularon restando la segunda de la primera en cada VD: cuatro subescalas del ACE (atención, memoria, lenguaje y fluencia verbal) y el Test Token. Dado que cada VD es una capacidad positiva, se denomina ganancia a cada una de estas diferencias.

Se utilizó el coeficiente de correlación lineal $r$ de Pearson para explorar las relaciones de los valores absolutos y ganancias (diferencias post - pre intervención) de las VD entre sí y con edad, años de escolaridad y puntaje en la escala Hachinski. Para testear la ausencia de diferencias iniciales, según se reciban o no alguna de las cinco formas de estimulación, se realizaron pruebas $t$ de Student sobre diferencias de medias independientes, con la corrección de Bonferroni para edad, género, años de estudio y las cinco VD.

Los cambios en los estados de las VD (cuatro subescalas del ACE: atención, memoria, lenguaje y fluencia verbal, y el Test Token) fueron testeados por separado mediante un análisis de covarianza (ANCOVA) para medidas repetidas (en dos tiempos), según las formas de estimulación que significan cada una de las cinco VI y sus interacciones. Los dos tiempos fueron los de las mediciones pre y post tratamiento para la variable activa: sesiones de RC. Los dos tiempos para las otras VI se tomaron mediando un lapso semejante al anterior, de entre 6 y 12 meses. Las cinco VI fueron todas dicotómicas según los participantes realizaran o no ejercicios físicos (gimnasia y/o caminatas), hobbies o actividades creativas, actividades mentales domiciliarias (crucigramas, sudoku, sopas de letras, entre otras), trabajo remunerado y participación en las sesiones de RC. El ANCOVA incluyó dos variables: puntaje en la Escala de Isquemia Hachinski y años de la escolaridad. La significación de la interacción entre las ganancias y cada una de las VI se evaluó con el estadístico $F$ multivariado. En el análisis de datos se usaron $d$ de Cohen y $\eta^{2}$ parcial como indicadores del tamaño del efecto.

Todos los análisis se realizaron con el programa SPSS.24.

\section{Resultados}

\section{Medidas Pretratamiento y Ganancias}

Tanto los valores pretratamiento de las VD como los de sus ganancias muestran que estos se encuentran moderada y directamente correlacionados entre sí $(p<0,001)$. Las correlaciones entre las medidas pretratamiento van desde $r=0,392$ entre ACE-fluencia verbal y ACE-lenguaje hasta $r=0,613$ entre ACE-lenguaje y TOKEN-comprensión verbal. Las correlaciones entre las ganancias van desde $r=0,346$ entre ACE-atención y TOKEN-comprensión verbal hasta $r=0,671$ entre ACE-memoria y ACE-fluencia verbal.

Los valores absolutos de las VD y de las ganancias para cada una de las formas de estimulación se exhiben en la Tabla 2.

En la Tabla 2 se aprecia que quienes desarrollaban al inicio actividades físicas presentan mayores valores en todas las VD, menos en atención. También se observa que quienes tenían actividad laboral presentan mayores valores iniciales en todas las VD, aunque debe recordarse que quienes participan en estas actividades tienden a tener menor edad. Por su parte, quienes recibieron la RC inicialmente no diferían en ninguna VD de quienes no la recibieron. 
Tabla 2

Estadísticos Descriptivos de las Variables Dependientes Pretratamiento y Ganancias según las Cinco Formas de Estimulación

\begin{tabular}{|c|c|c|c|c|c|c|c|c|c|c|c|c|}
\hline \multirow{3}{*}{ Estimulació } & & \multirow{3}{*}{ Estadístico } & \multicolumn{5}{|c|}{ VD Pretratamiento } & \multicolumn{5}{|c|}{ VD Ganancias (Post - Pretratamiento) } \\
\hline & & & \multicolumn{4}{|c|}{$\mathrm{ACE}$} & \multirow{2}{*}{$\frac{\text { TOKEN }}{\begin{array}{c}\text { Comprensión } \\
\text { verbal }\end{array}}$} & \multicolumn{4}{|c|}{$\mathrm{ACE}$} & \multirow{2}{*}{ 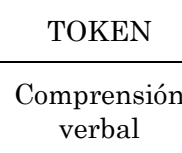 } \\
\hline & & & Atención & Memoria & Lenguaje & $\begin{array}{c}\text { Fluencia } \\
\text { verbal }\end{array}$ & & Atención & Memoria & Lenguaje & $\begin{array}{c}\text { Fluencia } \\
\text { verbal }\end{array}$ & \\
\hline \multirow{3}{*}{$\begin{array}{c}\text { Gimnasia y/o } \\
\text { caminatas }\end{array}$} & \multirow{2}{*}{ No } & Media & 7,4 & 21,7 & 24,2 & 5,8 & 73,4 & $-0,6$ & 0,2 & $-0,4$ & 0,4 & 1,2 \\
\hline & & $D E$ & 1,1 & 6,8 & 4,0 & 2,5 & 17,6 & 0,9 & 3,6 & 2,7 & 1,7 & 8,3 \\
\hline & Sí & $\begin{array}{l}\text { Media } \\
D E\end{array}$ & $\begin{array}{l}7,6 \\
0,9\end{array}$ & $\begin{array}{c}25,8^{*} \\
3,9\end{array}$ & $\begin{array}{c}25,6^{*} \\
2,9\end{array}$ & $\begin{array}{l}7,2^{*} \\
2,6\end{array}$ & $\begin{array}{l}80,2^{*} \\
15,9\end{array}$ & $\begin{array}{r}-0,3 \\
0,8\end{array}$ & $\begin{array}{r}-0,3 \\
3,0\end{array}$ & $\begin{array}{l}0,1 \\
3,4\end{array}$ & $\begin{array}{l}0,1 \\
2,2\end{array}$ & $\begin{array}{l}0,4 \\
6,3\end{array}$ \\
\hline \multirow{4}{*}{$\begin{array}{l}\text { Actividad } \\
\text { creativa }\end{array}$} & \multirow{2}{*}{ No } & Media & 7,4 & 22,9 & 24,6 & 6,2 & 75,1 & $-0,5$ & $-0,3$ & $-0,6$ & 0,1 & 0,4 \\
\hline & & $D E$ & 1,2 & 6,3 & 3,9 & 2,8 & 18,5 & 0,8 & 3,7 & 3,0 & 2,0 & 7,6 \\
\hline & \multirow{2}{*}{ Sí } & Media & 7,7 & 23,8 & 24,9 & 6,3 & 77,3 & $-0,5$ & 0,7 & $0,7^{*}$ & 0,7 & 2,3 \\
\hline & & $D E$ & 0,6 & 6,2 & 3,2 & 2,2 & 13,9 & 0,9 & 2,4 & 2,7 & 1,6 & 7,7 \\
\hline \multirow{4}{*}{$\begin{array}{l}\text { Crucigrama, } \\
\text { sudoku, sopa } \\
\text { de letras }\end{array}$} & \multirow{2}{*}{ No } & Media & 7,5 & 22,9 & 24,6 & 6,2 & 75,4 & $-0,6$ & $-0,5$ & $-0,5$ & $-0,1$ & 0,4 \\
\hline & & $D E$ & 1,0 & 6,3 & 3,9 & 2,7 & 17,9 & 0,9 & 3,3 & 2,9 & 1,5 & 8,1 \\
\hline & \multirow{2}{*}{ Sí } & Media & 7,3 & 24,2 & 25,4 & 6,3 & 77,1 & $0,0^{*}$ & $2,2^{*}$ & $1,1^{*}$ & $2,1^{*}$ & 3,6 \\
\hline & & $D E$ & 1,3 & 6,1 & 3,0 & 2,6 & 14,6 & 0,6 & 3,0 & 3,0 & 2,4 & 4,4 \\
\hline \multirow{4}{*}{ Trabaja } & \multirow{2}{*}{ No } & Media & 7,3 & 21,7 & 23,3 & 5,3 & 70,5 & $-0,6$ & 0,3 & 0,3 & 0,6 & $2,5^{*}$ \\
\hline & & $D E$ & 1,3 & 7,2 & 4,8 & 2,5 & 20,1 & 1,0 & 3,6 & 3,6 & 2,0 & 8,8 \\
\hline & \multirow{2}{*}{ Sí } & Media & $7,6^{*}$ & $24,2^{*}$ & $25,7^{*}$ & $6,9^{*}$ & $79,6^{*}$ & $-0,5$ & $-0,2$ & $-0,6$ & 0,0 & $-0,3$ \\
\hline & & $D E$ & 0,8 & 5,2 & 2,2 & 2,6 & 13,7 & 0,7 & 3,2 & 2,4 & 1,8 & 6,5 \\
\hline \multirow{4}{*}{ Sesiones RC } & \multirow{2}{*}{ No } & Media & 7,3 & 23,3 & 24,6 & 6,4 & 75,3 & $-0,8$ & $-1,6$ & $-1,0$ & $-0,7$ & $-1,9$ \\
\hline & & $D E$ & 1,2 & 6,4 & 4,2 & 2,8 & 18,0 & 0,8 & 2,5 & 2,7 & 1,3 & 6,1 \\
\hline & \multirow[t]{2}{*}{ Sí } & Media & 7,7 & 22,8 & 24,9 & 6,0 & 76,5 & $0,0^{*}$ & $2,8^{*}$ & $1,2^{*}$ & $1,9^{*}$ & $6,1^{*}$ \\
\hline & & $D E$ & 0,7 & 6,0 & 2,6 & 2,3 & 16,1 & 0,7 & 3,0 & 3,0 & 1,6 & 7,5 \\
\hline
\end{tabular}

Se utilizó la corrección de Bonferroni para todas las comparaciones por pares dentro de una columna. 
Sin embargo, la RC es la única forma de estimulación que presenta ganancias en las cinco VD. La realización de crucigramas, sudoku o sopa de letras presenta ganancias en cuatro VD. La actividad creativa muestra ganancia en el lenguaje y los que no trabajaban presentan ganancia en comprensión verbal (Test Token). Las otras formas de estimulación no se asociaron a ganancias.

Estos resultados son prometedores. Pero como las distintas formas de estimulación se han distribuido de forma natural en la muestra, incluso con superposiciones, convendría intentar alguna forma de control estadístico para aumentar la validez interna del estudio, mediante un método que permita tanto el control de sus interacciones como de terceras variables. En este sentido, dado que edad, años de escolaridad y el avance del deterioro vascular son teóricamente relevantes, se exploraron sus correlaciones con las VD cognitivas. Estos resultados tienen un valor intrínseco y también sirven para orientar la inclusión de variables a controlar en modelos multivariados, tanto en sus medidas pretratamiento como en sus ganancias.

Edad y años de escolaridad tuvieron una correlación inversa entre sí, $r=-0,29(p<0,001)$, lo que era esperable, por cuanto las personas mayores vivieron tiempos en donde la tendencia a prolongar los estudios era menor. Los años de escolaridad presentaron correlaciones significativas, directas, entre leves y moderadas, con los valores pretratamiento de las cinco VD: ACE-atención $(r=0,182)$, ACE-memoria $(r=0,234)$, ACElenguaje $(r=0,221)$, ACE-fluencia verbal $(r=0,502)$, TOKEN-comprensión verbal $(r=0,293)$ (en todos los casos $p<0,04)$. Dado lo anterior, se comprende que la edad haya presentado correlaciones inversas con los valores pretratamiento de cuatro de las VD, tres del ACE, memoria $(r=-0,390)$, lenguaje $(r=-0,243)$ y fluencia verbal $(r=-0,403)$ y también con Token-comprensión verbal $(r=-0,245)$; en todos los casos $p<0,006$. Sin embargo, aunque la edad no se correlacionó con ninguna ganancia, los años de escolaridad sí lo hicieron, inversa y levemente, con ganancias en tres VD: ACE-lenguaje $(r=-0,20)$, ACE-fluencia verbal $(r=-0,294)$ y Token-comprensión verbal $(r=-0,232)$; en todos los casos $p<0,03$, lo que mostraría una mayor capacidad explicativa de la escolaridad sobre la edad, dentro de un campo en buena medida compartido.

Por su parte, los puntajes en la escala de isquemia de Hachinski, que no presentaron ninguna correlación con las VD en su nivel pretratamiento, sí lo hicieron débilmente con las ganancias en dos variables: ACElenguaje y ACE-fluencia verbal ( $r=0,253, p<0,004$ y $r=0,198, p<0,025$, respectivamente).

Además, edades menores y mayores niveles de escolaridad son predictores de mejor nivel inicial en casi todas las VD. En contrapartida, las mayores ganancias se registraron en las funciones específicamente lingüísticas, entre quienes tienen mayor nivel de isquemia y, sobre todo, entre quienes han tenido una trayectoria académica más breve. Estos análisis sucesivos sugirieron la inclusión de los años de educación formal y el puntaje en la Escala de Isquemia de Hachinski como covariables del ANCOVA.

\section{Resultado Principal}

La Tabla 3 resume los resultados obtenidos mediante ANCOVA para cada una de las ganancias en las VD asociadas a cada forma de estimulación, independientemente de las otras y controlando los años de educación formal y el puntaje en la escala de Hachinski. El estadístico $F$ multivariado para los efectos de cada VI sobre las diferencias (ganancias) intrasujetos indica que la forma de estímulo que produce ganancias en mayor cantidad de las VD son las sesiones de RC, modificando (con $p<0,005$ ) directamente tres VD del ACE: atención, memoria y fluencia verbal, siendo en los tres casos su $\eta^{2}$ parcial $=0,09$.

La realización de actividades mentales y las actividades físicas son las otras formas de estimulación que evidencian cierto efecto positivo sobre las ganancias, pero lo hacen solo sobre ACE-fluencia verbal, que, a su vez, es la única VD en aparecer modificada por más de un factor. Por su parte, las actividades creativas (hobbies) y las actividades laborales no se asociaron a ninguna variación positiva en ganancias en VD. Por último, la consideración de la diferencia media intersujetos para el mismo ANCOVA (ver Tabla 4) según los niveles de cada forma de estimulación, refuerza las conclusiones del análisis principal en favor de las sesiones de RC. 
Tabla 3

Prueba ANCOVA de Medidas Repetidas para las Interacciones de Tiempo x Ganancias según las Cinco Formas de Estimulación*

\begin{tabular}{|c|c|c|c|c|c|c|c|c|c|c|c|c|c|c|c|c|c|c|c|c|c|c|}
\hline \multirow{2}{*}{$\begin{array}{l}\text { Ganancia } \\
\text { (post - pre) }\end{array}$} & \multirow[b]{2}{*}{ Cat. } & \multirow[b]{2}{*}{$n$} & \multicolumn{4}{|c|}{ ACE-atención } & \multicolumn{4}{|c|}{ ACE-memoria } & \multicolumn{4}{|c|}{ ACE-lenguaje } & \multicolumn{4}{|c|}{ ACE-fluencia verbal } & \multicolumn{4}{|c|}{ TOKEN-comprensión verbal } \\
\hline & & & Media & $D E$ & $F$ & $\begin{array}{c}p\left(\mathrm{n}^{2}\right. \\
\text { parcial })\end{array}$ & Media & $D E$ & $F$ & $\begin{array}{c}p\left(\mathrm{n}^{2}\right. \\
\text { parcial })\end{array}$ & Media & $D E$ & $F$ & $\begin{array}{c}p\left(\eta^{2}\right. \\
\text { parcial })\end{array}$ & Media & $D E$ & $F$ & $\begin{array}{c}p\left(\eta^{2}\right. \\
\text { parcial })\end{array}$ & Media & $D E$ & $F$ & $\begin{array}{c}p\left(\eta^{2}\right. \\
\text { parcial })\end{array}$ \\
\hline \multirow{2}{*}{$\begin{array}{l}\text { Gimnasia y/o } \\
\text { caminatas }\end{array}$} & No & 84 & $-0,58$ & 0,88 & \multirow{2}{*}{0,41} & \multirow{2}{*}{0,840} & 0,19 & 3,63 & \multirow[t]{2}{*}{0,03} & \multirow[b]{2}{*}{0,857} & $-0,40$ & 2,76 & \multirow{2}{*}{0,37} & \multirow{2}{*}{0,542} & 0,39 & 1,76 & \multirow{2}{*}{8,99} & \multirow{2}{*}{$\begin{array}{c}\mathbf{0 , 0 0 3} \\
(0,082)\end{array}$} & 1,24 & 8,32 & \multirow[t]{2}{*}{0,00} & \multirow{2}{*}{0,968} \\
\hline & Sí & 44 & $-0,34$ & 0,78 & & & $-0,34$ & 2,98 & & & 0,14 & 3,39 & & & 0,05 & 2,16 & & & 0,36 & 6,30 & & \\
\hline \multirow{2}{*}{$\begin{array}{l}\text { Actividad } \\
\text { creativa }\end{array}$} & No & 91 & $-0,53$ & 0,85 & \multirow{2}{*}{2,20} & \multirow{2}{*}{0,141} & $-0,30$ & 3,71 & \multirow[t]{2}{*}{0,00} & \multirow{2}{*}{0,982} & $-0,59$ & 3,02 & \multirow{2}{*}{2,47} & \multirow{2}{*}{0,119} & 0,10 & 1,97 & \multirow{2}{*}{2,54} & \multirow{2}{*}{0,114} & 0,35 & 7,60 & \multirow[t]{2}{*}{0,62} & \multirow{2}{*}{0,434} \\
\hline & Sí & 37 & $-0,43$ & 0,87 & & & 0,76 & 2,44 & & & 0,70 & 2,75 & & & 0,70 & 1,66 & & & 2,38 & 7,77 & & \\
\hline \multirow{2}{*}{$\begin{array}{l}\text { Crucigrama, } \\
\text { sudoku, sopa } \\
\text { de letras }\end{array}$} & No & 106 & $-0,60$ & 0,86 & & & $-0,44$ & 3,34 & 0,90 & & $-0,50$ & 2,93 & & & $-0,11$ & 1,55 & & $<0,001$ & 0,38 & 8,10 & 0,06 & \\
\hline & Sí & 22 & 0,00 & 0,62 & 0,07 & 0,788 & 2,18 & 2,97 & & 0,345 & 10,14 & 3,00 & 1,95 & 0,165 & 2,14 & 2,36 & 32,62 & $(0,246)$ & 3,64 & 4,37 & & 0,806 \\
\hline Traboig & No & 55 & $-0,55$ & 1,02 & 036 & 0,548 & 0,31 & 3,63 & 1,93 & 0168 & 0,31 & 3,62 & & 400 & 0,64 & 2,01 & & 0501 & 2,53 & 8,79 & 3,14 & \\
\hline Irabaja & Sí & 73 & $-0,47$ & 0,71 & 0,36 & 0,048 & $-0,22$ & 3,25 & & 0,168 & $-0,62$ & 2,37 & 0,48 & 0,490 & 0,00 & 1,78 & 0,46 & 0,501 & $-0,26$ & 6,52 & & 0,079 \\
\hline & No & 82 & $-0,78$ & 0,82 & & 0,003 & $-1,57$ & 2,48 & & 0,002 & $-0,99$ & 2,71 & & & $-0,66$ & 1,33 & & 0,002 & $-1,94$ & 6,15 & & \\
\hline Sesiones RC & Sí & 46 & 0,00 & 0,67 & 9,43 & $(0,086)$ & 2,83 & 3,03 & 9,88 & $(0,090)$ & 10,15 & 3,01 & 0,13 & 0,716 & 1,93 & 1,62 & 10,22 & $(0,093)$ & 6,07 & 7,50 & 3,34 & 0,071 \\
\hline Total & & 128 & $-0,50$ & 0,85 & & & 0,01 & 3,41 & & & $-0,22$ & 2,99 & & & 0,27 & 1,90 & & & 0,94 & 7,67 & & \\
\hline
\end{tabular}

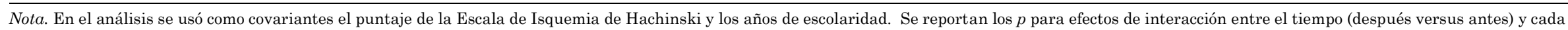
factor. Las $p<0,05$ están en negrita. 
Tabla 4

Prueba ANCOVA de Medidas Repetidas para las Ganancias según las Cinco Formas de Estimulación. Pruebas de los Efectos Intersujetos

\begin{tabular}{|c|c|c|c|c|c|c|c|c|c|c|c|c|c|c|c|c|c|c|c|c|}
\hline \multirow{2}{*}{$\begin{array}{c}\text { Ganancia } \\
\text { VI }\end{array}$} & \multicolumn{4}{|c|}{ ACE-atención } & \multicolumn{4}{|c|}{ ACE-memoria } & \multicolumn{4}{|c|}{ ACE-lenguaje } & \multicolumn{4}{|c|}{ ACE-fluencia verbal } & \multicolumn{4}{|c|}{ TOKEN-comprensión verbal } \\
\hline & $F$ & $p$ & $\begin{array}{c}\eta^{2} \\
\text { parcial }\end{array}$ & $\begin{array}{l}\text { Potencia } \\
\text { (obs) }\end{array}$ & $F$ & $p$ & $\begin{array}{c}\eta^{2} \\
\text { parcial }\end{array}$ & $\begin{array}{c}\text { Potencia } \\
\quad \text { (obs) }\end{array}$ & $F$ & $p$ & $\begin{array}{c}\eta^{2} \\
\text { parcial }\end{array}$ & $\begin{array}{c}\text { Potencia } \\
\text { (obs) }\end{array}$ & $F$ & $p$ & $\begin{array}{c}\eta^{2} \\
\text { parcial }\end{array}$ & $\begin{array}{l}\text { Potencia } \\
\text { (obs) }\end{array}$ & $F$ & $p$ & $\begin{array}{c}\eta^{2} \\
\text { parcial }\end{array}$ & $\begin{array}{l}\text { Potencia } \\
\text { (obs) }\end{array}$ \\
\hline Intersección & 154,00 & $<0,001$ & 0,607 & 1,000 & 36,72 & $<0,001$ & 0,269 & 1,000 & 143,00 & $<0,001$ & 0,588 & 1,000 & 9,44 & 0,003 & 0,086 & 0,861 & 73,79 & $<0,001$ & 0,425 & 1,000 \\
\hline Educación & 3,39 & 0,069 & & 0,446 & 2,05 & 0,155 & & 0,295 & 1,67 & 0,199 & & 0,249 & 19,54 & $<0,001$ & 0,163 & 0,992 & 5,96 & 0,016 & 0,056 & 0,677 \\
\hline $\begin{array}{l}\text { Escala de } \\
\text { Hachinski_T }\end{array}$ & 0,17 & 0,681 & & 0,069 & 0,38 & 0,541 & & 0,093 & 0,37 & 0,545 & & 0,092 & 0,07 & 0,790 & & 0,058 & 1,93 & 0,168 & & 0,279 \\
\hline $\begin{array}{l}\text { Actividad } \\
\text { física }\end{array}$ & 0,97 & 0,327 & & 0,164 & 2,72 & 0,102 & & 0,373 & 3,69 & 0,057 & & 0,477 & 1,07 & 0,303 & & 0,176 & 3,20 & 0,077 & & 0,425 \\
\hline Hobbies & 0,51 & 0,475 & & 0,109 & 0,10 & 0,752 & & 0,061 & 0,54 & 0,463 & & 0,113 & 0,33 & 0,569 & & 0,087 & 0,16 & 0,686 & & 0,069 \\
\hline $\begin{array}{l}\text { Actividad } \\
\text { mental }\end{array}$ & 0,04 & 0,834 & & 0,055 & 0,62 & 0,435 & & 0,121 & 0,81 & 0,369 & & 0,145 & 0,58 & 0,448 & & 0,117 & 0,01 & 0,923 & & 0,051 \\
\hline $\begin{array}{l}\text { Actividad } \\
\text { laboral }\end{array}$ & 0,10 & 0,749 & & 0,062 & 0,14 & 0,705 & & 0,066 & 1,42 & 0,236 & & 0,219 & 0,00 & 0,995 & & 0,050 & 2,58 & 0,111 & & 0,357 \\
\hline Sesiones RC & 8,21 & 0,005 & 0,076 & 0,810 & 2,62 & 0,109 & & 0,361 & 4,20 & 0,043 & 0,040 & 0,528 & 6,34 & 0,013 & 0,060 & 0,703 & 7,05 & 0,009 & 0,066 & 0,748 \\
\hline
\end{tabular}

Nota. Sólo se reportan efectos principales. Se resaltan en negrita los $p<0,05$. 
Para ilustrar estos conceptos se pueden considerar las representaciones de las evoluciones desde estos niveles de una VD, en este caso ACE-memoria. Aunque no existe una diferencia significativa entre ambos puntajes, la Figura 1 muestra una tendencia a una ganancia mínima entre quienes trabajan, pero pareciera mayor entre quienes no trabajan.

\section{Figura 1}

Puntajes de ACE-Memoria Pre y Post Intervención, según Realización de Actividad Laboral

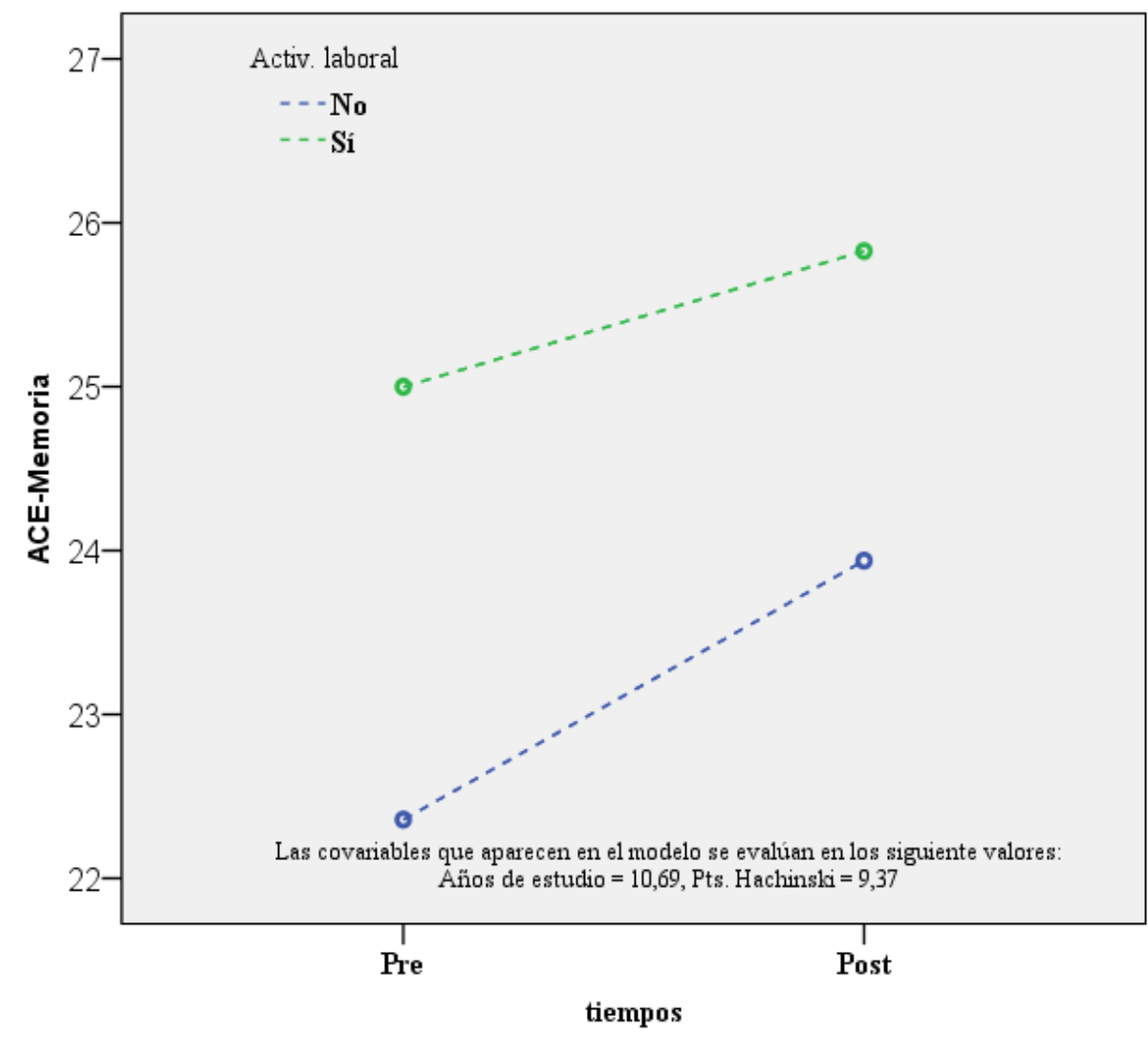

Nota. Los puntajes de ACE-memoria están ajustados por años de estudio y el puntaje en la Escala de Isquemia de Hachisnki. No trabaja: $n=55$; Sí trabaja: $n=73$.

Esas tendencias se invierten en la Figura 2, donde, la comparación se hace entre los que efectúan actividades mentales domiciliarias (crucigramas, sudoku, sopa de letras y otras), aunque las diferencias entre las ganancias en ACE-memoria de quienes no realizan estas actividades mentales y de quienes sí tampoco resulten estadísticamente significativas. 


\section{Figura 2}

Puntajes de ACE-Memoria Pre y Post Intervención, según Realización de Actividades Mentales Domiciliarias

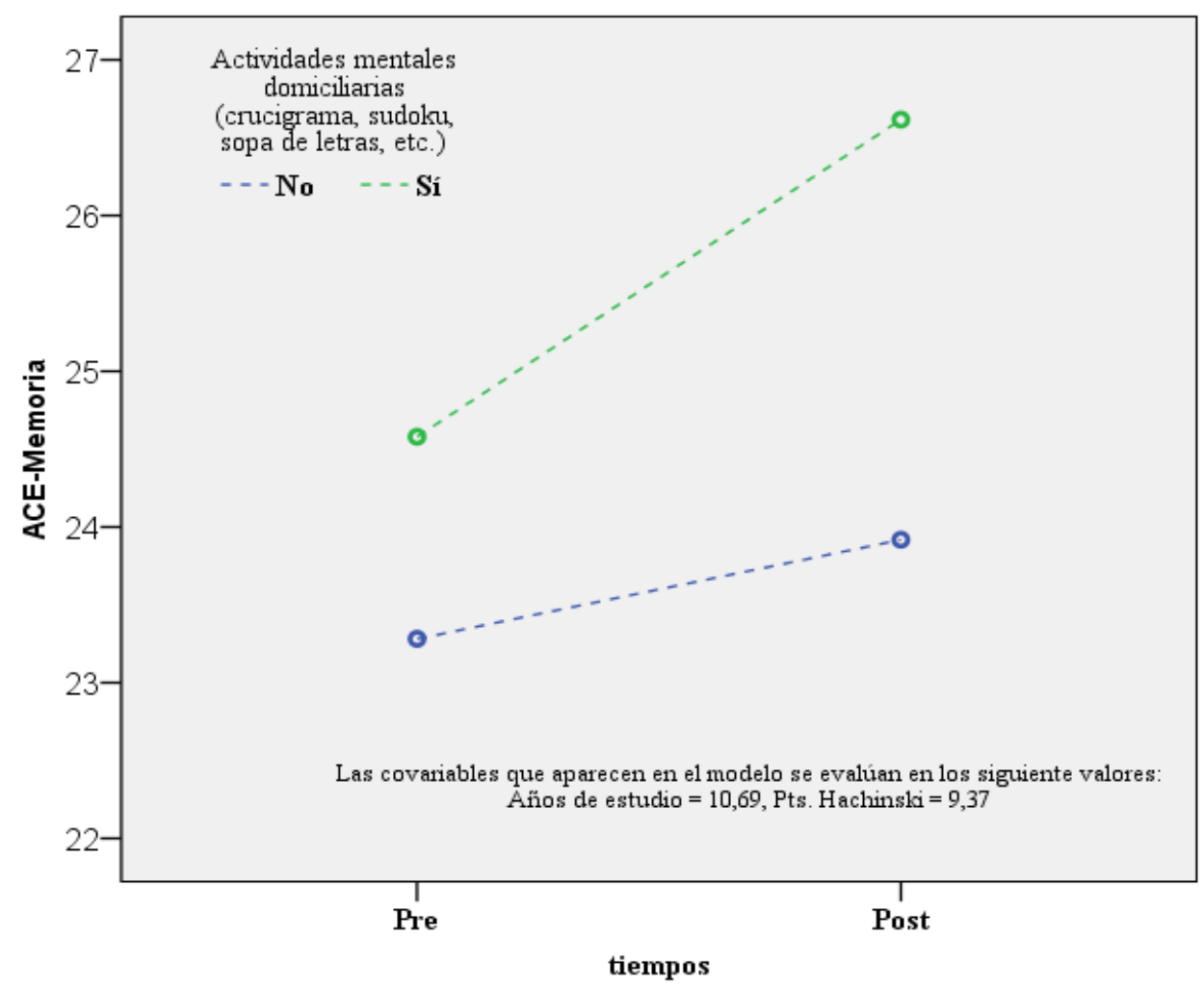

Nota. Los puntajes de ACE-memoria están ajustados por años de estudio y el puntaje en la Escala de Isquemia de Hachisnki. No realiza actividades mentales domiciliarias: $n=106$; Sí realiza actividades mentales domiciliarias: $n=22$.

En cambio, la diferencia en la evolución aparece nítida en la Figura 3, donde se comparan las ganancias en ACE-memoria según la concurrencia a sesiones de RC. Allí no solo aparecen ganancias claras en quienes recibieron $\mathrm{RC}$, sino que los que no concurrieron muestran leves pérdidas. 


\section{Figura 3}

Puntajes de ACE-Memoria Pre y Post Intervención, según Realización de Sesiones de Rehabilitación Cognitiva

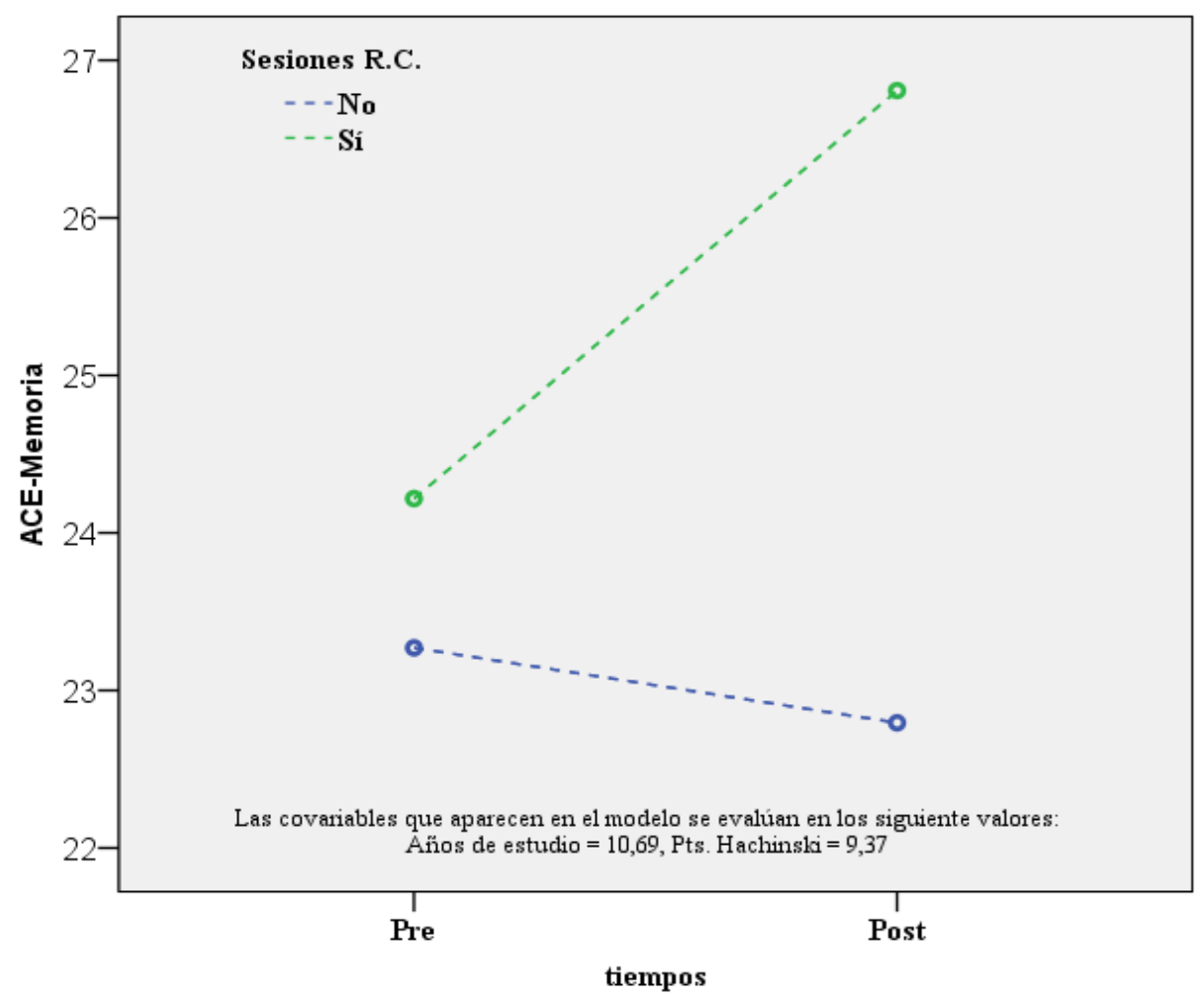

Nota. Los puntajes de ACE-memoria están ajustados por años de estudio y el puntaje en la Escala de Isquemia de Hachisnki. No realiza sesiones de RC: $n=82$; Sí realiza sesiones de RC: $n=46$.

\section{Discusión}

En primer lugar, resulta clara la conclusión general del presente estudio: en los límites de esta investigación y para la población estudiada, la $\mathrm{RC}$ es la forma de estimulación que supera a las otras consideradas en la capacidad de obtener ganancias, o al menos evitar pérdidas, en varias funciones cognitivas y, por lo tanto, la prioritariamente recomendable para esta población.

Establecido ello, quedan múltiples interrogantes e hipótesis en torno a la cuestión:

- Si se interrumpe la RC ¿qué pasa con las ganancias obtenidas más allá de los 6 meses no cubiertos por los datos? ¿Se pierden, mantienen o aumentan?

- Si se continúa la RC ¿existe un tope en cuanto a ganancia? ¿se mantienen crecientes al menos las ventajas contra la ausencia de RC? Naturalmente, resolver estos interrogantes, requeriría más mediciones de seguimiento a lo largo de más tiempo y con un mayor tamaño muestral.

- Aun dentro del lapso establecido, quedaría por determinar cómo se da la evolución de las ganancias: ¿aumentando al inicio? ¿aumentando al final? ¿progreso homogéneo? La respuesta a esta interrogante daría indicios para la respuesta de la primera serie de preguntas.

- La merma inicial detectada en las subpruebas y su modificación posterior luego al periodo de RC ¿está relacionada con la capacidad del cerebro de adaptarse y cambiar como resultado de la estimulación, tal como postula Stern (2003)?

- Se estudiaron aquí cinco variables y en cuatro se registró un impacto diferencial de la RC. En ellas hay inicialmente puntajes por debajo de lo esperable, tomando como base los dominios cognitivos del test de cribado ACE. La RC muestra asociación con mejoras en áreas de memoria, lenguaje y atención. ¿Podría estar indicando una remisión al estado anterior de la memoria global, iniciando por la función atencional que se va prologando en el tiempo, tal como sugirió la impresión clínica? 
- Más ampliamente: las moderadas correlaciones entre esas cinco variables, tanto en el tiempo pre y aún en el tiempo postintervención señalan un comportamiento correlacionado de las cinco funciones consideradas. La existencia de estas correlaciones no permite establecer con claridad si hay alguna/s de ellas/s que sufren el impacto diferencial y "arrastra/n" a las restantes o si la intervención impacta directa y conjuntamente en todas ellas o si hubiese efecto en un factor subyacente del cual ellas serían emergentes.

- ¿Hay otras dimensiones y variables cognitivas, no directamente lingüísticas, en las que las sesiones de RC pueden mostrar un efecto semejante?

- ¿Cuáles son los elementos de una estimulación compleja y multimodal, como el dispositivo de RC implementado, que producen las mayores ganancias? Por ejemplo, podría hipotetizarse que los resultados obtenidos se deben a ejercicios cognitivos efectuados en PC. Estos están relacionados específicamente con intervención del lenguaje desde actividades en PC e implican la participación de otras funciones en vista a operaciones de escritura y lectura, en las que colabora la memoria global. También es posible que, en sesiones de RC la influencia del profesional interviniente a través de dinámicas personalizadas y del intercambio con el paciente, dentro de una estrategia personalizada y adaptada, modifique otras variables no estudiadas.

- ¿Cómo interactúan los elementos específicos de la RC con los factores comunes (vínculo terapéutico, motivación, autoeficacia, seguimiento, autonomía, autosatisfacción, seguimiento personalizado, entre otros) a otros dispositivos de estimulación?

- Ligado a lo anterior ¿cómo potenciar y diversificar el efecto de un dispositivo de RC a otras funciones cognitivas?

- Superando la cuestión básica de las funciones cognitivas ¿cuánto y cómo de estas ganancias se trasladan a la vida cotidiana?

En segundo lugar, las actividades mentales domiciliarias (como crucigrama, sudoku o sopa de letras) se mostraron asociadas a ciertas ganancias. Lamentablemente, para esta submuestra los participantes que realizaban estas actividades eran 22 casos, 13 de los cuales también asistieron a sesiones de RC, y la forma de operacionalización (por autorreporte) de la submuestra sugieren ser más cautos con la conclusión sobre su efectividad. De todos modos, este tipo de actividades quedan sugeridas, sea por su capacidad de ser una dimensión en un dispositivo multimodal de estimulación como el aquí propuesto, sea por consistir en un medio económico de estimulación preventiva, focalizada o aun universal.

En tercer lugar, también hay que ser cauto en torno a la incapacidad de mostrar ganancias de tres tipos de actividades incluidas en este estudio: actividades creativas tipo hobbies, actividades físicas y actividades laborales. Como se ha dicho, ellas cuentan con cierto aval teórico y algún respaldo empírico y, si bien en esta comparación resultan inefectivas, no necesariamente se podría inferir su falta de efectividad. Tal cautela estriba en dos series de razones. La primera de ellas es de carácter metodológico: los valores de estas variables aquí están dados por autorreporte con una sola medición, lo que hace que su precisión y confiabilidad sean hasta cierto punto cuestionables. La segunda serie de razones es que, por el carácter rutinario y no manipulado, estas formas de estimulación habrían estado ejerciendo su influjo durante largo tiempo antes del comienzo del estudio, por lo que no contarían con la ventaja de resultar estímulos novedosos o disruptivos, ventaja que sí tienen las dos formas de estimulación con ganancias aquí probadas (sesiones de RC y actividades domiciliarias de refuerzo cognitivo). También podría pensarse que, en el momento del inicio del estudio, justamente por ser rutinarias, ya habrían "agotado" su efecto benéfico en quienes las reciben.

En cuarto y último lugar, la distinción dentro del constructo RCog es propuesta como reserva cognitiva restante (RCR). La RCR denomina a lo que resta de la RCog, esta reserva existente en el momento de la instalación del DCL y se va gastado parcialmente en su empleo por vías de alternativas o compensaciones desarrolladas en actividades de la vida cotidiana.

La distinción de la RCR permite dilucidar dos hechos que no encontrarían clara explicación en el marco teórico. En primer lugar, la mencionada ausencia de resultados positivos vinculados a supuestas fuentes rutinarias o estables de estimulación (actividades físicas, creativas o laborales) al cabo de seis meses. Ello se interpreta desde el efecto que tuvieron las fuentes rutinarias durante largo tiempo, pero que ya no producen ganancias medibles. Las fuentes rutinarias fueron agotando la RCog y la RCR es baja. En segundo lugar, si bien la edad y el nivel de escolaridad tienen correlaciones inversas y directas, respectivamente, con casi todas las VD en sus estados iniciales, las ganancias se correlacionan inversamente con la duración de la trayectoria académica y, en menor medida y en forma directa, con la intensidad del deterioro. 
Se puede resumir y superar lo anterior si se hipotetiza que, frente al DCL con base vascular ya instalado, las personas usan naturalmente su RCog y, al hacerlo, les va quedando una menor RCR. Esta RCR sería el potencial de recuperación o de ralentización o tope del avance del DC, actualizable a través de estímulos artificiales consistentes, sistemáticos y novedosos, como los que propone la $\mathrm{RC}$, de tal modo que se activa a medida que se registran déficits entre los estímulos o desafíos cognitivos y el rendimiento cognitivo habitual. Para usar una imagen, el RCR sería como lo que queda de una batería eléctrica auxiliar (la RCog) que ha venido suplementando con su flujo las mermas de una batería principal cuando, por alguna falla, el flujo de esta ha sido incapaz de cubrir las exigencias del sistema eléctrico al que proveía. La RCR, más que la RCog, sería el blanco actual de las sesiones de RC. El potencial de la RCog tiene una limitación: solo puede suplir los déficits del sistema principal hasta cierto punto. Esto explicaría por qué los rendimientos iniciales en personas naturalmente estimuladas tienden a ser superiores: ellas ya vienen recurriendo a la RCog. También explicaría por qué para quienes han recibido menos estimulaciones, por haber tenido una trayectoria académica más breve y una consecuente demanda inferior de actividad mental en la vida cotidiana, la RCog, que venía siendo subutilizada, tiene más prestaciones por dar, es decir, tiene mayor RCR.

Conclusiones: La RC tiene como objetivo mejorar funciones cognitivas como la memoria, atención y lenguaje, entre otras posibles. Los resultados del estudio muestran que las sesiones de RC con eventuales refuerzos domiciliarios mediante actividades escritas o en PC (por plataforma) y, en segundo lugar, los ejercicios de refuerzo cognitivo domiciliarios son las formas de intervención con mejores resultados, habilitando ganancias y favoreciendo la remisión, el mantenimiento y aun la recuperación parcial de funciones cognitivas en pacientes con DCL, comprobado mediante test, y con base de hipertensión.

Otro aspecto descrito se refiere a la continuidad en el beneficio o ganancia que permitió inicialmente una notable mejora persistente a lo largo de los meses, aun cuando existe aumento de edad y las consecuentes modificaciones morfológicas, biológicas y fisiológicas. Este suceso normal de envejecimiento, aun con HTA, no fue impedimento para la obtención de ganancias asociadas a algunas formas de estimulación.

Por lo antes dicho, es importante que, luego del neurodiagnóstico, los pacientes y sus familias sean adecuadamente informados de los objetivos, tratamientos disponibles, del sentido de las estrategias a seguir, de las ganancias esperables en términos de recuperación o, al menos, de ralentización o tope del avance del DC, en función de la RCR y en pos de beneficios en su calidad de vida. De esa manera, se podrá lograr una mayor adherencia y mejores resultados.

\section{Referencias}

American Psychiatric Association. (2014). Manual diagnóstico y estadístico de los trastornos mentales: DSM-5 (5ª ed.). Editorial Médica Panamericana.

Arenaza-Urquijo, E. M., Bosch, B., Sala-Llonch, R., Solé-Padullés, C., Junqué, C., Fernández-Espejo, D., Bargalló, N., Rami, L., Molinuevo, J. L. \& Bartrés-Faz, D. (2011). Specific anatomic associations between white matter integrity and cognitive reserve in normal and cognitively impaired elders. The American Journal of Geriatric Psychiatry, 19(1), 33-42. https://doi.org/10.1097/JGP.0b013e3181e448e1

Arriola Manchola, E., Carnero Pardo, C., Freire Pérez, A., López Mongil, R., López Trigo, J. A., Manzano Palomo, S. \& Olazarán Rodríguez, J. (2017). Deterioro cognitivo leve en el adulto mayor (Documento de consenso). Sociedad Española de Geriatría y Gerontología. https://www.segg.es/media/descargas/Consenso\%20deteriorocognitivoleve.pdf

Arroyo-Anlló, E. M., Poveda Díaz-Marta, J. \& Chamorro Sánchez, J. (2012). Técnicas de rehabilitación neuropsicológica en demencias: hacia la ciberrehabilitación neuropsicológica. Pensamiento Psicológico, 10(1), 107-127. http://www.scielo.org.co/scielo.php?script=sci arttext\&pid=S165789612012000100008

Bartoloni, L. C. (2019). Deterioro cognitivo. Diagnosis, 16(1), Artículo 2. https://www.revistadiagnosis.org.ar/index.php/diagnosis/article/view/227 Bruna, O., Roig, T., Puyuelo, M., Junqué, C. \& Ruano, Á. (2011). Rehabilitación neuropsicológica: intervención y práctica clínica. Elsevier Masson.

Calero Morales, S., Klever Díaz, T., Caiza Cumbajin, M. R., Rodríguez Torres, Á. F. \& Analuiza Analuiza, E. F. (2016). Influencia de las actividades físico-recreativas en la autoestima del adulto mayor. Revista Cubana de Investigaciones Biomédicas, 35(4), 366-374. http://www.dspace.uce.edu.ec/bitstream/25000/14809/3/Influencia\%20de\%20las\%20actividades\%20f\%C3\%ADsicorecreativas\%20en\%20la\%20autoestima\%20del\%20adulto\%20mayor.pdf

Campbell, N. L., Unverzagt, F., LaMantia, M. A., Khan, B. A. \& Boustani, M. A. (2013). Risk factors for the progression of mild cognitive impairment to dementia. Clinics in Geriatric Medicine, 29(4), 873-893. https://doi.org/10.1016/j.cger.2013.07.009

Cancino M. \& Rehbein, L. (2016). Factores de riesgo y precursores del deterioro cognitivo leve (DCL): una mirada sinóptica. Terapia Psicológica, 34(3), 183-189. https://doi.org/10.4067/S0718-48082016000300002

De Renzi, E. \& Faglioni, P. (1978). Normative data and screening power of a shortened version of the Token Test. Cortex, 14(1), 41-49. https://doi.org/10.1016/S0010-9452(78)80006-9

De Renzi, E. \& Vignolo, L. A. (1962). Token Test: A sensitive test to detect receptive disturbances in aphasics. Brain, 85(4), 665-678. https://doi.org/10.1093/brain/85.4.665 
Diaz, J. F., Merskey, H., Hachinski, V. C., Lee, D. H., Boniferro, M., Wong, C. J., Mirsen, T. R. \& Fox, H. (1991). Improved recognition of leukoaraiosis and cognitive impairment in Alzheimer's disease. Archives of Neurology, 48, $1022-1025$. https://doi.org/10.1001/archneur.1991.00530220038016

División de Población. (2014). La situación demográfica en el mundo: informe conciso. Secretaría de las Naciones Unidas, Departamento de Asuntos Económicos $\quad$ y http://www.un.org/en/development/desa/population/publications/pdf/trends/Concise\%20Report\%20on\%20the \%20World\%20Popula tion\%20Situation\%202014/es.pdf

Forlenza, O. V., Diniz, B. S., Stella, F., Teixeira, A. L. \& Gattaz, W. F. (2013). Mild cognitive impairment (Part 1): Clinical characteristics and predictors of dementia. Revista Brasileira de Psiquiatria, 35(2), 178-185. https://doi.org/10.1590/1516-4446-2012-3503

García-Caballero, A., García-Lado, I., González-Hermida, J., Recimil, M. J., Area, R., Manes, F., Lamas, S. \& Berrios, G.E. (2006). Validation of the Spanish version of the Addenbrooke's Cognitive Examination in a rural community in Spain. International Journal of Geriatric Psychiatry, 21(3), 239-245. https://doi.org/10.1002/gps.1450

Garre-Olmo, J. (2018). Epidemiología de la enfermedad de Alzheimer y otras demencias. Revista de Neurología de España, 66(11), 377386. https://doi.org/10.33588/rn.6611.2017519

Hachinski, V., Iadecola, C., Petersen, R. C., Breteler, M. M., Nyenhuis, D. L., Black, S. E., Powers, W. J., DeCarli, C., Merino, J. G., Kalaria, R. N., Vinters, H. V., Holtzman, D. M., Rosenberg, G. A., Wallin, A., Dichgans, M., Marler, J. R., Leblanc, G. G. (2006). National Institute of Neurological Disorders and Stroke-Canadian stroke network vascular cognitive impairment harmonization standards. Stroke, 37(9), 2220-2241. https://doi.org/10.1161/01.STR.0000237236.88823.47

Hachinski, V. C., Iliff, L. D., Zilhka, E., Du Boulay, G. H., McAllister, V. L., Marshall, J., Ross Russell, R. W. \& Symon, L. (1975). Cerebral blood flow in dementia. Archives of Neurology, 32(9), 632-637. https://doi.org/10.1001/archneur.1975.00490510088009

Hernández Silvera, D. I. (2009). Enhebrando huellas: un quehacer gerontológico desde la teoría del encuentro. San Pablo.

Hernando-Requejo, V. (2016). Nutrición y deterioro cognitivo. Nutrición Hospitalaria, 33(Supl. 4), 49-52. https://doi.org/10.20960/nh.346

Lojo-Seoane, C., Facal, D. \& Juncos-Rabadán, O. (2012). ¿Previene la actividad intelectual el deterioro cognitivo? Relaciones entre reserva cognitiva y deterioro cognitivo ligero. Revista Española de Geriatría y Gerontología, 47(6), $270-278$. https://doi.org/10.1016/j.regg.2012.02.006

Luque, L. E. \& Gonzalez Verheust, M. C. (2012). Eficacia de un programa de estimulación cognitiva en adultos mayores sanos. En Facultad de Psicología, Memorias IV Congreso Internacional de Investigación y Práctica Profesional en Psicología, XIX Jornadas de Investigación y VIII Encuentro de Investigadores en Psicología del Mercosur, Noviembre 27-30, 2012, Psicología del Desarrollo Tomo 3, Trabajos Libres (pp. 90-93). Universidad de Buenos Aires. http://jimemorias.psi.uba.ar/index.aspx?anio=2012

Martínez de la Iglesia, J., Onís Vilches, M. C., Dueñas Herrero, R., Albert Colomer, C., Aguado Taberné, C. \& Luque Luque, R. (2002). Versión española del cuestionario de Yesavage abreviado (GDS) para el despistaje de depresión en mayores de 65 años: adaptación y validación. Medicina Familiar y Comunitaria, 12(10), 620-630. https://doi.org/10.4321/S1131-57682002001000003

Mathuranath, P. S., Nestor, P. J., Berrios, G. E., Rakowicz, W. \& Hodges, J. R. (2000). A brief cognitive test battery to differentiate Alzheimer's disease and frontotemporal dementia. Neurology, 55(11), 1613-1620. https://doi.org/10.1212/01.wnl.0000434309.85312.19

Meléndez-Moral, J. C., Sanz-Álvarez, T. \& Navarro-Pardo, E. (2012). Deterioro cognitivo leve: método y procedimiento de clasificación. Anales de Psicología, 28(2), 604-610. https://doi.org/10.6018/analesps.28.2.148891

Nevado Rey, M. (2017). Intervenciones no farmacológicas. Revista Española de Geriatría y Gerontología, 52(Supl. 1), 44-46. https://doi.org/10.1016/S0211-139X(18)30080-5

Pantoni, L. \& Inzitari, D. (1993). Hachinski's ischemic score and the diagnosis of vascular dementia: A review. Italian Journal of Neurological Sciences, 14, 539-546. https://doi.org/10.1007/bf02339212

Pohjasvaara, T., Mäntylä, R., Ylikoski, R., Kaste, M. \& Erkinjuntti, T. (2000). Comparison of different clinical criteria (DSM-III, ADDTC, ICD10, NINDS-AIREN, DSM-IV) for the diagnosis of vascular dementia. Stroke, 31(12), 2952-2957. https://doi.org/10.1161/01.STR.31.12.2952

Portillo Barrera, P. (2015). Factores de riesgo para el deterioro cognitivo leve en adultos mayores de Maracaibo [Tesis de doctorado, Universidad Autónoma de Madrid]. Repositorio Universidad Autónoma de Madrid. http://hdl.handle.net/10486/670960

Rodríguez García, P. L. \& Rodríguez García, D. (2015). Diagnóstico del deterioro cognitivo vascular y sus principales categorías. Neurología, 30(4), 223-239. https://doi.org/10.1016/j.nrl.2011.12.014

Sarasola, D., Luján-Calcagno, M. d., Sabe, L., Crivelli, L., Torralva, T., Roca, M., García-Caballero, A. \& Manes, F. (2005). El Addenbrooke's Cognitive Examination en español para el diagnóstico de demencia y para la diferenciación entre enfermedad de Alzheimer y demencia frontotemporal. Revista de Neurología, 41(2), 717-721. https://doi.org/10.33588/rn.4112.2004625

Schapira, M., Cohen, G., Guajardo, M. E., Martinez, D., Schapira, M., Seinhart, B. D. \& Soderlund, M. E. (2020). Reflexiones sobre vivir con demencia en épocas del COVID-2019. Revista Argentina de Gerontología y Geriatría, 34(1), 19-20. https://ringofox.agency/sagg/wp-content/uploads/2021/09/RAGG_04_2020-19-20.pdf

Serrano, C. M., Taragano, F., Allegri, R. F., Krupitzki, H., Martelli, M., Feldman, M., Goscilo, C., Tufró, G., Loñ, L., Sarasola, D., Dillon, C. \& Tamaroff, L. (2007). Factores predictores de conversión en deterioro cognitivo leve. (Cohorte de Seguimiento en CEMIC). Revista Neurológica Argentina, 32(2), 75-93. http://nueva.cemic.edu.ar/descargas/2007\%20MCI\%20Cohorte\%20(H).pdf

Souza, L. \& Vivas, L. (2017). Valores normativos del Addenbrooke's Cognitive Examination (ACE) para población con bajo nivel socioeducativo. Neurología Argentina, 9(4), 219-224. https://doi.org/10.1016/j.neuarg.2017.07.005

Spellacy, F. J. \& Spreen, O. (1969). A short form of the Token Test. Cortex, 5(4), 390-397. https://doi.org/10.1016/S0010-9452(69)80015-8

Stern, Y. (2002). What is cognitive reserve? Theory and research application of the reserve concept. Journal of the International Neuropsychological Society, 8(3), 448-460. https://doi.org/10.1017/S1355617702813248

Stern, Y. (2003). The concept of cognitive reserve: A catalyst for research. Journal of Clinical and Experimental Neuropsychology, 25(5), 589-593. https://doi.org/10.1076/jcen.25.5.589.14571

Thomas Carazo, E. \& Nadal Blanco, M. J. (2001). Abordaje diagnóstico y terapéutico de la demencia en atención primaria. Medicina de Familia-SEMERGEN, 27(11), 575-586. https://doi.org/10.1016/S1138-3593(01)74030-0

van Harskamp, F. \& van Dongen, H. R. (1977). Construction and validation of different short forms of the Token Test. Neuropsychologia, 15(3), 467-470. https://doi.org/10.1016/0028-3932(77)90100-2

Ye, S., Dong, S., Tan, J., Chen, L., Yang, H., Chen, Y., Peng, Z., Huo, Y., Liu, J., Tang, M., Li, Y., Zhou, H. \& Tao, Y. (2019). Whitematter hyperintensities and lacunar infarcts are associated with an increased risk of Alzheimer's disease in the elderly in China. Journal of Clinical Neurology, 15(1), 46-53. https://doi.org/10.3988/jcn.2019.15.1.46 
Yesavage, J. A. \& Sheikh, J. I. (1986). Geriatric Depression Scale (GDS): Recent evidence and development of a shorter version. Clinical Gerontologist, 5(1-2), 165-173. https://doi.org/10.1300/j018v05n01_09

Fecha de recepción: Febrero de 2020.

Fecha de aceptación: Mayo de 2021. 